\title{
Structural Stability of Discontinuous Galerkin Schemes
}

\section{Acta Applicandae}

\section{Mathematicae}

An International Research

Journal on Applying

Mathematics and Mathematical

Applications

\section{ISSN 0167-8019}

Volume 113

Number 1

Acta Appl Math (2010)

113:45-56

DOI $10.1007 /$

s10440-010-9583-5

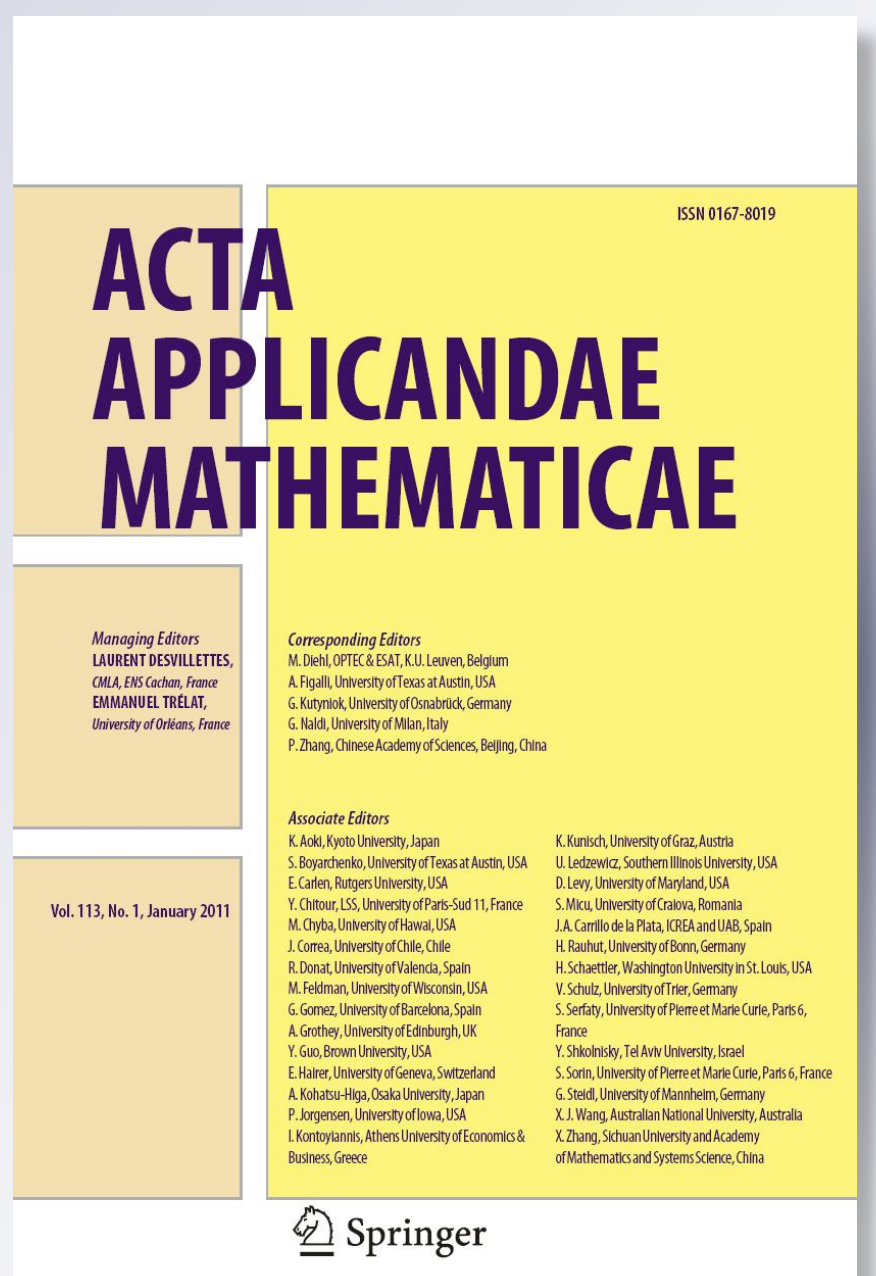

Springer 
Your article is protected by copyright and all rights are held exclusively by Springer Science+Business Media B.V.. This e-offprint is for personal use only and shall not be selfarchived in electronic repositories. If you wish to self-archive your work, please use the accepted author's version for posting to your own website or your institution's repository. You may further deposit the accepted author's version on a funder's repository at a funder's request, provided it is not made publicly available until 12 months after publication. 


\title{
Structural Stability of Discontinuous Galerkin Schemes
}

\author{
Claire David · Pierre Sagaut
}

Received: 6 June 2010 / Accepted: 30 July 2010 / Published online: 18 August 2010

(C) Springer Science+Business Media B.V. 2010

\begin{abstract}
The goal of this work is to determine classes of traveling solitary wave solutions for a differential approximation of a discontinuous Galerkin finite difference scheme by means of an hyperbolic ansatz. It is shown that spurious solitary waves can occur in finitedifference solutions of nonlinear wave equation. The occurence of such a spurious solitary wave, which exhibits a very long life time, results in a non-vanishing numerical error for arbitrary time in unbounded numerical domain. Such a behavior is referred here to have a structural instability of the scheme, since the space of solutions spanned by the numerical scheme encompasses types of solutions (solitary waves in the present case) that are not solutions of the original continuous equations. This paper extends our previous work about classical schemes to discontinuous Galerkin schemes (David and Sagaut in Chaos Solitons Fractals 41(4):2193-2199, 2009; Chaos Solitons Fractals 41(2):655-660, 2009).
\end{abstract}

Keywords Discontinuous Galerkin method $\cdot$ Solitary waves $\cdot$ Numerical flux $\cdot$ Structural stability

Mathematics Subject Classification (2000) $65 \mathrm{M} 06 \cdot 65 \mathrm{M} 12 \cdot 65 \mathrm{M} 60 \cdot 35 \mathrm{~B} 99$

\section{Introduction: The Discontinuous Galerkin Method}

The discontinuous Galerkin $(D G)$ methods are very popular ones that enable one to solve partial differential equations.

The discontinuous Galerkin method was introduced by Reed and Hill [3] for the problem of neutron transport. LeSaint and Raviart [4] analysed the method in this context and proved a rate of convergence of $\mathrm{O}$ for smooth solutions on Cartesian grids. A number of researchers have made significant contributions since then. Among others, Lin and Zhou [5-7] proved convergence of the method for nonsmooth solutions. Moreover, Cockburn and Shu [8-10]

C. David $(\bowtie) \cdot$ P. Sagaut

Université Pierre et Marie Curie-Paris 6, Institut Jean Le Rond d'Alembert, UMR CNRS 7190,

Boîte courrier 162, 4 place Jussieu, 75252 Paris cedex 05, France

e-mail: Claire.David@upmc.fr 
analysed and extended the original discontinuous Galerkin method to systems of hyperbolic conservation laws and convection-dominated problems.

The higher order $D G$ method has been applied to nonlinear hyperbolic conservation laws as early as in 1989 by Cockburn and Shu [8]. Afterwards the $D G$ method enjoys a rapid development, see, e.g. Cockburn et al. [8-10], Feistauer et al. [11], Houston, Suli et al. [12] and the references therein. The $D G$ methods compromise the ideas of numerical fluxes and limiters into a framework of finite element methods. Like all finite element methods, the $D G$ methods can handle complex geometries and incorporate naturally boundary conditions. Important practical advantage of the $D G$ methods in comparison with the finite volume-type schemes is the fact that the $D G$ methods have more compact stencils than the finite volume methods.

\section{Weak Formulation}

The discontinuous Galerkin method can be applied to any equation of the form:

$$
\frac{\partial u}{\partial t}+\nabla \cdot \vec{F}(u)=0
$$

where $\vec{F}$ is a flux vector, function of the conservative state vector $u$, on a domain divided into arbitrarily shaped elements $\Omega_{i}$ that cover the domain.

An initial condition, $U_{0}(x)=U(x ; t=0)$, and appropriate boundary conditions must also be given.

One of the most used case concerns the unsteady, compressible Euler equations.

The discretization in a discontinuous Galerkin method starts with a variational formulation as in a standard Galerkin method, but allows for discontinuities over the element edges.

To get the variational formulation of (1), we partition the domain into a collection of nonoverlapping elements. The $D G$ method is defined by choosing a set of local basis functions $\varphi_{i}^{l}$ for each $\Omega_{i}$, such that the solution in $\Omega_{i}$ can be approximated as:

$$
u_{i}=\sum_{l=1}^{N} u_{i}^{l} \varphi_{i}^{l}
$$

On any $\Omega_{i}$ :

$$
\frac{\partial u_{i}}{\partial t}+\nabla \cdot \vec{F}\left(u_{i}\right)=0
$$

Projection onto each member of the basis set yields:

$$
\left(\varphi_{i}^{l}, \frac{\partial u_{i}}{\partial t}\right)+\left(\varphi_{i}^{l}, \nabla \cdot \vec{F}\left(u_{i}\right)\right)=0
$$

Integration on $\Omega_{i}$ leads to:

$$
\int_{\Omega_{i}}\left(\varphi_{i}^{l}, \frac{\partial u_{i}}{\partial t}\right) d \Omega+\int_{\Omega_{i}}\left(\varphi_{i}^{l}, \nabla \cdot \vec{F}\left(u_{i}\right)\right) d \Omega=0
$$

and:

$$
\int_{\Omega_{i}}\left(\varphi_{i}^{l}, \frac{\partial u_{i}}{\partial t}\right) d \Omega-\int_{\Omega_{i}}\left(\nabla \varphi_{i}^{l}, \vec{F}\left(u_{i}\right)\right) d \Omega+\int_{\partial \Omega_{i}}\left(\varphi_{i}^{l}, \vec{F}^{R}\right) n_{i} d s=0
$$


where $\partial \Omega_{i}$ is the segment of the element boundary that is common to the neighboring element $\Omega_{j}, n_{i}$ is the unit outward-normal vector on $\partial \Omega_{i}$.

$\vec{F}^{R}$ denotes a numerical flux which is usually an approximate Riemann flux of the LaxFriedrichs or other type.

The coordinates $u_{i}^{l}$ of the approximate solution $u_{i}$ are the new unknowns.

Since each element has a distinct local approximate solution,the solution on each interior edge is double valued and discontinuous. The approximate Riemann flux $\vec{F}^{R}$ resolves the discontinuity and provides the only mechanism by which adjacent elements communicate. Due to the fact that this communication occurs in an edge integral means the solution in a given element $\Omega_{i}$ depends only on the edge trace of the neighboring solution $U_{i}$, and not on the whole of the neighboring solution $U_{j, \Omega_{j}}$ connex to $\Omega_{i}$.

The $D G$ method is efficiently implemented on general unstructured grids to any order of accuracy using the quadrature-free formulation.

In the quadrature-free formulation, developed by Atkins and Shu in [13], the flux vector $\vec{F}$ is approximated in terms of the basis set $\varphi_{i}^{l}$, and the approximate Riemann flux $\vec{F}^{R}$ is approximated in terms of the lower basis set $\psi_{i}^{l}$ :

$$
\begin{gathered}
\vec{F}=\sum_{l=1}^{N} \overrightarrow{f_{i}^{l}} \varphi_{i}^{l} \\
\vec{F}^{R}=\sum_{l=1}^{N} f^{R^{l}} \psi_{i}^{l}
\end{gathered}
$$

We have:

$$
\begin{gathered}
\int_{\Omega_{i}}\left(\varphi_{i}^{l}, \frac{\partial\left\{\sum_{k=1}^{N} u_{i}^{k} \varphi_{i}^{k}\right\}}{\partial t}\right) d \Omega-\int_{\Omega_{i}}\left(\nabla \varphi_{i}^{l},\left\{\sum_{k=1}^{N} f_{i}^{k} \varphi_{i}^{k}\right\}\right) d \Omega \\
+\int_{\partial \Omega_{i}}\left(\varphi_{i}^{l},\left\{\sum_{k=1}^{N} f_{i}^{R_{i}^{k}} \psi_{i}^{k}\right\}\right) n_{i} d s=0
\end{gathered}
$$

i.e.:

$$
\sum_{k=1}^{N}\left\{\int_{\Omega_{i}}\left(\varphi_{i}^{l}, \frac{\partial\left\{u_{i}^{k} \varphi_{i}^{k}\right\}}{\partial t}\right) d \Omega-\int_{\Omega_{i}}\left(\nabla \varphi_{i}^{l}, f_{i}^{k} \varphi_{i}^{k}\right) d \Omega+\int_{\partial \Omega_{i}}\left(\varphi_{i}^{l}, f^{R_{i}^{k}} \psi_{i}^{k}\right) n_{i} d s\right\}=0
$$

With these approximations, the volume and boundary integrals can be evaluated analytically, instead of by quadrature, leading to a simple sequence of matrix-vector operations:

$$
\frac{\partial u_{i}^{l}}{\partial t}=\left(M^{-1} A\right)\left[f_{i}^{l}\right]-\sum_{l=1}^{N}\left(M^{-1} B_{i j}\right)\left[f_{i}^{R^{l}}\right]
$$

where:

$$
\begin{aligned}
M & =\left[\int_{\Omega}\left(\varphi_{i}^{l}, \varphi_{i}^{k}\right) d \Omega\right] \\
A & =\left[\int_{\Omega}\left(\nabla \varphi_{i}^{l}, \varphi_{i}^{k}\right) d \Omega\right]
\end{aligned}
$$




$$
B=\left[\int_{\partial \Omega}\left(\nabla \varphi_{i}^{l}, \psi_{i}^{k}\right) d s\right]
$$

The matrices $M, A$, and $B$ depend only on the shape of the similarity element and the degree $p$ of the solution.

In order to obtain fully discrete $D G$ methods, a suitable time discretization has to be applied. Since the above described numerical schemes, finite volume methods and finite volume evolution Galerkin (FVEG) schemes are explicit in time, we consider here only time explicit $D G$ schemes, too. Since the Runge-Kutta discretization is explicit in time, a $c f$ stability condition has to be imposed to guarantee the stability of the scheme.

\section{The One-Dimensional Burgers Case}

In the following, we consider the one-dimensional scalar conservation law:

$$
u_{t}+[f(u)]_{x}=0, \quad u(x, 0)=u_{0}(x)
$$

where $f$ denotes a function of $u$.

The one-dimensional cells are denoted by $I_{i}=\left[x_{i-\frac{1}{2}}, x_{i+\frac{1}{2}}\right]$, and their centers by $x_{i}=$ $\frac{1}{2}\left\{x_{i-\frac{1}{2}}+x_{i+\frac{1}{2}}\right\}$.

A temporal Taylor expansion at the $k$ th order yields:

$$
u(x, t+\tau)=u(x, t)+\sum_{l=1}^{k} \frac{\tau^{l}}{l !} u_{t, l}+o\left(\tau^{k}\right)
$$

The temporal derivatives terms in the above expansion can be replaced by spatial ones using (15):

$$
\left\{\begin{array}{l}
u_{t}=-[f(u)]_{x}=-f^{\prime}(u) u_{x} \\
u_{t t}=-\left[f^{\prime}(u) u_{x}\right]_{t}=-f^{\prime \prime}(u) u_{x} u_{t}-f^{\prime}(u) u_{x t} \\
\ldots
\end{array}\right.
$$

At the third order:

$$
u(x, t+\tau)=u(x, t)-\tau F_{x}
$$

where:

$$
\left\{\begin{array}{l}
F=f+f^{*} \\
f^{*}=\frac{\tau}{2} f^{\prime}(u) u_{t}+\frac{\tau^{2}}{6}\left\{f^{\prime \prime}(u) u_{t}^{2}+f^{\prime}(u) u_{t t}\right\}
\end{array}\right.
$$

An important aspect of the $D G$ discretization is the choice of an appropriate set of basis functions, which are polynomials ones.

Although $D G$ solutions do not rely on the choice of basis, the computational efficiency of the $D G$ scheme depends on the basis. For instance, choosing the set of Legendre polynomials of degree up to $N$ as the basis set will lead to a diagonal mass matrix, because of the $L^{2}$ orthogonality of the Legendre polynomials. This is all the more interesting as explicit time integration can be performed without inverting or lumping the mass matrix.

An alternative approach is to use a nodal basis, where the basis set is constructed using Lagrange-Legendre polynomials with roots at Gauss-Lobatto quadrature points [17]. 
In the following, the $D G$ solution as well as the test function space is given by

$$
\mathcal{V}_{a}^{k}=\left\{P / P_{\mid I_{i}} \in \mathcal{P}^{k}\left(I_{i}\right)\right\}
$$

where $\mathcal{P}^{k}\left(I_{i}\right)$ denotes the space of polynomials of degree $\leqslant k$ on the cell $I_{i}$.

Its is convenient to retain the normalized Legendre polynomials, which constitute a local orthogonal basis over $I_{i}$ :

$$
P_{l}^{i}(x)=\sqrt{\frac{2 l+1}{2}} \frac{1}{2^{l} l !} \frac{d^{l}\left[\left(\xi^{2}-1\right)^{l}\right]}{d \xi^{l}}, \quad l=0, \ldots, k
$$

In the one-dimensional coordinate system, the test functions as well as the approximate solution $U_{a}$ are expanded in terms of tensor product functions from $\mathcal{V}_{a}^{k}$ :

$$
U_{a}(x, t)=\sum_{l=0}^{N} U_{i}^{l} P_{l}^{i}(x), \quad x \in I_{i}
$$

Thus, the degrees of freedom are the moments $U_{i}^{l}$ defined by:

$$
U_{i}^{l}=\int_{I_{i}} U_{a}(x, t) P_{l}^{i}(x) d x
$$

The scheme (15) reads then:

$$
U_{i}^{l}\left(t^{n+1}\right)=U_{i}^{l}\left(t^{n+1}\right)-\int_{I_{i}} F P_{l x}^{i}(x) d x+\hat{F}_{i+\frac{1}{2}} P_{l}^{i}\left(x_{i+\frac{1}{2}}\right)-\hat{F}_{i-\frac{1}{2}} P_{l}^{i}\left(x_{i-\frac{1}{2}}\right)
$$

where $\hat{F}_{i+\frac{1}{2}}$ is a numerical flux which depends on the values of the numerical solution and its spatial derivatives at the cell interfaces $x_{i-\frac{1}{2}}$ and $x_{i-\frac{1}{2}}$.

This numerical flux is related to generalized Riemann solvers.

In most of the $D G$ papers in the literature, the two point, first-order monotone LaxFriedrichs numerical flux is used due to its simplicity. However, there exist many other numerical fluxes based on various approximate Riemann solvers, such as the Godunov flux, the HLL flux [18], and a modification of the HLL flux, often referred to as the HLLC flux, ....

In the following, we consider the case of the Lax-Friedrichs and HLL fluxes, which, in our study, stand out as representative ones.

\subsection{The Lax-Friedrichs Flux}

The Lax-Friedrichs flux is defined by:

$$
\begin{aligned}
\hat{F}_{i+\frac{1}{2}} & =\frac{1}{2}\left\{F_{i-\frac{1}{2}}^{-}+F_{i-\frac{1}{2}}^{+}-\alpha\left(u_{i+\frac{1}{2}}^{+}-u_{i-\frac{1}{2}}^{-}\right)\right\} \\
& =\frac{1}{2}\left\{f_{i-\frac{1}{2}}^{-}+f_{i-\frac{1}{2}}^{+}-\alpha\left(u_{i+\frac{1}{2}}^{+}-u_{i-\frac{1}{2}}^{-}\right)\right\}+\frac{1}{2}\left(f_{i+\frac{1}{2}}^{*}-f_{i+\frac{1}{2}}^{*}+\right)
\end{aligned}
$$


where:

$$
\left\{\begin{array} { l } 
{ u _ { i + \frac { 1 } { 2 } } ^ { + } = \operatorname { l i m } _ { x \rightarrow x _ { i + \frac { 1 } { 2 } } , x > x _ { i + \frac { 1 } { 2 } } } u , } \\
{ u _ { i + \frac { 1 } { 2 } } ^ { - } = \operatorname { l i m } _ { x \rightarrow x _ { i + \frac { 1 } { 2 } } , x < x _ { i + \frac { 1 } { 2 } } } u , } \\
{ F _ { i + \frac { 1 } { 2 } } ^ { + } = \operatorname { l i m } _ { x \rightarrow x _ { i + \frac { 1 } { 2 } } , x > x _ { i + \frac { 1 } { 2 } } } u , } \\
{ F _ { i + \frac { 1 } { 2 } } ^ { - } = \operatorname { l i m } _ { x \rightarrow x _ { i + \frac { 1 } { 2 } } , x < x _ { i + \frac { 1 } { 2 } } } u , }
\end{array} \quad \left\{\begin{array}{l}
f_{i+\frac{1}{2}}^{+}=\lim _{x \rightarrow x_{i+\frac{1}{2}}, x>x_{i+\frac{1}{2}}} u \\
f_{i+\frac{1}{2}}^{-}=\lim _{x \rightarrow x_{i+\frac{1}{2}}, x<x_{i+\frac{1}{2}}} u \\
f_{i+\frac{1}{2}}^{*+}=\lim _{x \rightarrow x_{i+\frac{1}{2}}, x>x_{i+\frac{1}{2}}} f^{*} \\
f_{i+\frac{1}{2}}^{*-}=\lim _{x \rightarrow x_{i+\frac{1}{2}}, x<x_{i+\frac{1}{2}}} f^{*}
\end{array}\right.\right.
$$

and where:

$$
\alpha=\max _{u}\left|f^{\prime}(u)\right|
$$

(For the system case, the maximum is taken for the eigenvalues of the Jacobian $f^{\prime}(u)$.)

\subsection{The Harten-Lax-van Leer $(H L L)$ Flux}

The HLL flux [18] is based on the approximate Riemann solver with only three constant states separated by two waves.

In the following, we consider the one-dimensional system case of Euler equations for compressible gas dynamics:

$$
u=\left[\begin{array}{c}
\rho \\
\rho v \\
E
\end{array}\right], \quad f(u)=\left[\begin{array}{c}
\rho v \\
\rho v^{2}+p \\
v(E+p)
\end{array}\right]
$$

where $\rho$ is the density, $v$ the velocity, $E$ the total energy, $p$ the pressure, related to the total energy through:

$$
E=\frac{p}{\gamma-1}+\frac{1}{2} \rho v^{2}
$$

where $\gamma=1.4$ for air.

The HLL flux is then given by:

$$
\hat{f}_{u^{-}, u^{+}}^{H L L}= \begin{cases}f\left(u^{-}\right) & \text {if } 0 \leqslant s^{-} \\ F_{L}^{*}=\frac{s^{+} f\left(u^{-}\right)-s^{-} f\left(u^{+}\right)+s^{-} s^{+}\left(u^{+}-u^{-}\right)}{s^{+}-s^{-}} & \text {if } s^{-} \leqslant 0 \leqslant s^{+} \\ f\left(u^{+}\right) & \text {if } s^{+} \leqslant 0\end{cases}
$$

where the lower and upper bounds of the wave speed, $s^{-}$and $s^{+}+$, are estimated by means of the pressure-velocity estimates given by Toro [19]:

$$
\left\{\begin{array}{l}
s^{-}=v^{-}-c^{-} q^{-} \\
s^{*}=v^{*} \\
s^{+}=v^{+}-c^{+} q^{+}
\end{array}\right.
$$

where, for $K= \pm$ :

$$
q^{K}= \begin{cases}1 & \text { if } p^{-} \leqslant p^{K} \\ \sqrt{1+\frac{\gamma+1}{2 \gamma}\left(\frac{p^{*}}{p^{K}}-1\right)} & \text { if } p^{-}>p^{K}\end{cases}
$$


with:

$$
\left\{\begin{array}{l}
p^{*}=\frac{1}{2}\left(p^{-}+p^{+}\right)-\frac{1}{2}\left(v^{+}-v^{-}\right) \bar{\rho} \bar{c} \\
v^{*}=\frac{1}{2}\left(v^{-}+v^{+}\right)-\frac{p^{+}-p^{-}}{2 \bar{\rho} \bar{c}}\left(v^{+}-v^{-}\right)
\end{array}\right.
$$

where:

$$
\left\{\begin{array}{l}
\bar{\rho}=\frac{1}{2}\left(\rho^{-}+\rho^{+}\right) \\
\bar{c}=\frac{1}{2}\left(v^{-}+v^{+}\right)\left(c^{-}+c^{+}\right)
\end{array}\right.
$$

The final $H L L$ flux is given by:

$$
\hat{F}_{u^{-}, u^{+}}^{H L L}=\hat{f}_{u^{-}, u^{+}}^{H L L}+\frac{1}{2}\left\{f^{*}\left(u^{-}\right)+f^{*}\left(u^{+}\right)\right\}
$$

\section{Spurious Lattice Solitons}

The discrete solution associated with the $D G$ numerical scheme will admit spurious solitary waves, and therefore spurious local energy pile-up and local sudden growth of the error, if the discrete relation (24) is satisfied by a solitary wave.

Following [1, 2, 14-16, 20-25], we search solitary waves solution of (24) under the form:

$$
u(x, t, k)=A \operatorname{sech}[k(x-v t)]+B \tanh [k(x-v t)]
$$

\subsection{The Case of the Lax-Friedrichs Flux, for Burgers Equation}

In the case of the Burgers equation, the Lax-Friedrichs flux is given by:

$$
f(u)=u^{2}+\mu u_{x}
$$

where $\mu$ denotes the viscosity.

Using (36), the solution is searched, for each cell $I_{i}$, corresponding to $x=i h$, at the time $t=n \tau$, under the form:

$$
\left\{\begin{array}{l}
u_{i}=A_{i} \operatorname{sech}\left[k_{i}\left(i h-n v_{i} \tau\right)\right]+B_{i} \tanh \left[k_{i}\left(i h-n v_{i} \tau\right)\right] \\
u_{i-\frac{1}{2}}^{-}=A_{i-\frac{1}{2}}^{-} \operatorname{sech}\left[k_{i-\frac{1}{2}}^{-}\left(i h-v_{i-\frac{1}{2}}^{-} n \tau\right)\right]+B_{i-\frac{1}{2}}^{-} \tanh \left[k_{i-\frac{1}{2}}^{-}\left(i h-v_{i-\frac{1}{2}}^{-} n \tau\right)\right] \\
u_{i+\frac{1}{2}}^{-}=A_{i+\frac{1}{2}}^{+} \operatorname{sech}\left[k_{i+\frac{1}{2}}^{+}\left(i h-v_{i+\frac{1}{2}}^{+} n \tau\right)\right]+B_{i+\frac{1}{2}}^{+} \tanh \left[k_{i+\frac{1}{2}}^{+}\left(i h-v_{i+\frac{1}{2}}^{+} n \tau\right)\right]
\end{array}\right.
$$

where we have taken:

$$
u_{i}=u_{i-\frac{1}{2}}^{+}=u_{i+\frac{1}{2}}^{-}
$$

The constants $A_{i}, B_{i}, k_{i}, v_{i}, A_{i \pm \frac{1}{2}}^{ \pm}, B_{i \pm \frac{1}{2}}^{ \pm}, k_{i \pm \frac{1}{2}}^{ \pm}, v_{i \pm \frac{1}{2}}^{ \pm}$naturally depend on the $i$ th cell. Depending on their existence, and of the one of integers $i, n$ satisfying this relation, spurious lattice solitary waves will or not appear.

For sake of simplification, we set:

$$
\mathcal{E}=\left\{A_{i}, B_{i}, k_{i}, v_{i}, A_{i \pm \frac{1}{2}}^{ \pm}, B_{i \pm \frac{1}{2}}^{ \pm}, k_{i \pm \frac{1}{2}}^{ \pm}, v_{i \pm \frac{1}{2}}^{ \pm}\right\}
$$


Replacing (38), (25) in (24), for the first Legendre polynomial, in the specific case of kink profile solitary waves, for which the sech constants are taken equal to zero $\left(A_{i}^{ \pm}=A_{i \pm \frac{1}{2}}^{ \pm}=0\right)$, one obtains:

$$
B_{i+\frac{1}{2}}^{+}=-6 \alpha \tanh \left(k_{i+\frac{1}{2}}^{+}\left(1-j v_{i+\frac{1}{2}}^{+} \tau\right)\right) \mathcal{B}\left(\mathcal{E} \backslash B_{i+\frac{1}{2}}^{+}, C_{i}\right)
$$

where the function $\mathcal{B}$ is given by

$$
\begin{aligned}
& \mathcal{B}=-B_{i}^{4} k_{i}^{4} v_{i-\frac{1}{2}}^{-}{ }^{2} \tau^{2} \operatorname{sech}^{8}\left(k_{i}\left(-j v_{i+\frac{1}{2}}^{-} \tau-1\right)\right)-2 B_{i}^{3} k_{i}^{5} v_{i-\frac{1}{2}}^{-} \mu \tau^{2} \operatorname{sech}^{8}\left(k_{i}\left(-j v_{i-\frac{1}{2}}^{-} \tau-1\right)\right) \\
& +6 B_{i} k_{i} \mu \operatorname{sech}^{2}\left(k_{i}\left(-j v_{i-\frac{1}{2}}^{-} \tau-1\right)\right)-B_{i-\frac{1}{2}}^{-}{ }^{4} k_{i-\frac{1}{2}}^{-}{ }^{4} v_{i-\frac{1}{2}}^{-}{ }^{2} \tau^{2} \\
& +6 B_{i-\frac{1}{2}}^{-} k_{i-\frac{1}{2}}^{-} \mu \operatorname{sech}^{2}\left(k_{i+\frac{1}{2}}^{-}\left(-j v_{i-\frac{1}{2}}^{-} \tau-1\right)\right)+12 C_{i} \\
& -2 B_{i}^{4} k_{i}^{4} v_{i-\frac{1}{2}}^{-}{ }^{2} \tau^{2} \operatorname{sech}^{8}\left(k_{i}\left(1-j v_{i-\frac{1}{2}}^{-} \tau\right)\right)+12 B_{i} k_{i} \mu \operatorname{sech}^{2}\left(k_{i}\left(1-j v_{i-\frac{1}{2}}^{-} \tau\right)\right) \\
& -B_{i-\frac{1}{2}}^{-}{ }^{4} k_{i-\frac{1}{2}}^{-}{ }^{4} v_{i-\frac{1}{2}}^{-}{ }^{2} \tau^{2} \operatorname{sech}^{8}\left(k_{i-\frac{1}{2}}^{-}\left(-j v_{i-\frac{1}{2}}^{-} \tau-1\right)\right) \\
& -2 B_{i-\frac{1}{2}}^{-}{ }^{3} k_{i-\frac{1}{2}}^{-}{ }^{5} v_{i-\frac{1}{2}}^{-}{ }^{2} \mu \tau^{2} \operatorname{sech}^{8}\left(k_{i-\frac{1}{2}}^{-}\left(-j v_{i-\frac{1}{2}}^{-} \tau-1\right)\right) \\
& -4 B_{i}^{3} k_{i}^{5} v i l^{2} \mu \tau^{2} \operatorname{sech}\left[k i\left(1-j v_{i-\frac{1}{2}}^{-} \tau\right)\right]^{8}+6 B_{i} \alpha \tanh [k i(-1-j \operatorname{sech} \tau)] \\
& +3 B_{i}^{3} k_{i}^{2} v_{i-\frac{1}{2}}^{-} \tau \operatorname{sech}\left[k i\left(-1-j v_{i-\frac{1}{2}}^{-} \tau\right)\right]^{4} \tanh \left[k_{i}\left(-1-j v_{i-\frac{1}{2}}^{-} \tau\right)\right] \\
& +2 B_{i}^{3} k_{i}^{3} v_{i-\frac{1}{2}}^{-} \tau^{2} \tanh ^{2}\left(k_{i}\left(-j v_{i-\frac{1}{2}}^{-} \tau-1\right)\right) \operatorname{sech}^{4}\left(k_{i}\left(-j v_{i-\frac{1}{2}}^{-} \tau-1\right)\right) \\
& +6 B_{i}^{2} k_{i}^{3} v_{i-\frac{1}{2}}^{-} \mu \tau \tanh \left(k_{i}\left(-j v_{i-\frac{1}{2}}^{-} \tau-1\right)\right) \operatorname{sech}^{4}\left(k_{i}\left(-j v_{i-\frac{1}{2}}^{-} \tau-1\right)\right) \\
& -3 B_{i}^{2} \tanh ^{2}\left(k_{i}\left(-j v_{i-\frac{1}{2}}^{-} \tau-1\right)\right) \\
& -2 B_{i}^{4} k_{i}^{4} v_{i-\frac{1}{2}}^{-} \tau^{2} \tanh ^{2}\left(k_{i}\left(-j v_{i-\frac{1}{2}}^{-} \tau-1\right)\right) \operatorname{sech}^{6}\left(k_{i}\left(-j v_{i-\frac{1}{2}}^{-} \tau-1\right)\right) \\
& +4 B_{i}^{2} k_{i}^{4}{v_{i-\frac{1}{2}}^{-}}^{2} \mu \tau^{2} \tanh ^{2}\left(k_{i}\left(-j v_{i-\frac{1}{2}}^{-} \tau-1\right)\right) \operatorname{sech}^{4}\left(k_{i}\left(-j v_{i-\frac{1}{2}}^{-} \tau-1\right)\right) \\
& +4 B_{i}^{3} k_{i}^{5}{v_{i-\frac{1}{2}}^{-}}^{2} \mu \tau^{2} \tanh ^{2}\left(k_{i}\left(-j v_{i-\frac{1}{2}}^{-} \tau-1\right)\right) \operatorname{sech}^{6}\left(k_{i}\left(-j v_{i-\frac{1}{2}}^{-} \tau-1\right)\right) \\
& +3 B_{i-\frac{1}{2}}^{-}{ }^{3} k_{i-\frac{1}{2}}^{-}{ }^{2} v_{i-\frac{1}{2}}^{-} \tau \tanh \left(k_{i-\frac{1}{2}}^{-}\left(-j v_{i-\frac{1}{2}}^{-} \tau-1\right)\right) \operatorname{sech}^{4}\left(k_{i-\frac{1}{2}}^{-}\left(-j v_{i-\frac{1}{2}}^{-} \tau-1\right)\right) \\
& -6 B_{i-\frac{1}{2}}^{-} \alpha \tanh \left(k_{i-\frac{1}{2}}^{-}\left(-j v_{i-\frac{1}{2}}^{-} \tau-1\right)\right) \\
& +4 B_{i}^{3} k_{i}^{5} v_{i-\frac{1}{2}}^{-} \mu \tau^{2} \tanh ^{2}\left(k_{i}\left(-j v_{i-\frac{1}{2}}^{-} \tau-1\right)\right) \operatorname{sech}^{6}\left(k_{i}\left(-j v_{i-\frac{1}{2}}^{-} \tau-1\right)\right) \\
& +2 B_{i-\frac{1}{2}}^{-}{ }^{3} k_{i-\frac{1}{2}}^{-}{ }^{3} v_{i-\frac{1}{2}}^{-}{ }^{2} \tau^{2} \tanh ^{2}\left(k_{i-\frac{1}{2}}^{-}\left(-j v_{i-\frac{1}{2}}^{-} \tau-1\right)\right) \operatorname{sech}^{4}\left(k_{i-\frac{1}{2}}^{-}\left(-j v_{i-\frac{1}{2}}^{-} \tau-1\right)\right) \\
& +3 B_{i-\frac{1}{2}}^{-}{ }^{3} k_{i-\frac{1}{2}}^{-}{ }^{2} v_{i-\frac{1}{2}}^{-} \tau \tanh \left(k_{i-\frac{1}{2}}^{-}\left(-j v_{i-\frac{1}{2}}^{-} \tau-1\right)\right) \operatorname{sech}^{4}\left(k_{i-\frac{1}{2}}^{-}\left(-j v_{i-\frac{1}{2}}^{-} \tau-1\right)\right) \\
& +6 B_{i-\frac{1}{2}}^{-}{ }^{2} k_{i-\frac{1}{2}}^{-}{ }^{3} v_{i-\frac{1}{2}}^{-} \mu \tau \tanh \left(k_{i-\frac{1}{2}}^{-}\left(-j v_{i-\frac{1}{2}}^{-} \tau-1\right)\right) \operatorname{sech}^{4}\left(k_{i-\frac{1}{2}}^{-}\left(-j v_{i-\frac{1}{2}}^{-} \tau-1\right)\right)
\end{aligned}
$$




$$
\begin{aligned}
& -3 B_{i-\frac{1}{2}}^{-} \tanh ^{2}\left(k_{i-\frac{1}{2}}^{-}\left(-j v_{i-\frac{1}{2}}^{-} \tau-1\right)\right)-6 B_{i-\frac{1}{2}}^{-} \alpha \tanh \left(k_{i-\frac{1}{2}}^{-}\left(-j v_{i-\frac{1}{2}}^{-} \tau-1\right)\right) \\
& \times 2 B_{i-\frac{1}{2}}^{-}{ }^{4} k_{i-\frac{1}{2}}^{-}{ }^{4} v_{i-\frac{1}{2}}^{-}{ }^{2} \tau^{2} \tanh ^{2}\left(k_{i-\frac{1}{2}}^{-}\left(-j v_{i-\frac{1}{2}}^{-} \tau-1\right)\right) \operatorname{sech}^{6}\left(k_{i-\frac{1}{2}}^{-}\left(-j v_{i-\frac{1}{2}}^{-} \tau-1\right)\right) \\
& +4 B_{i-\frac{1}{2}}^{-}{ }^{2} k_{i-\frac{1}{2}}^{-}{ }^{4} v_{i-\frac{1}{2}}^{-}{ }^{2} \mu \tau^{2} \tanh ^{2}\left(k_{i-\frac{1}{2}}^{-}\left(-j v_{i-\frac{1}{2}}^{-} \tau-1\right)\right) \operatorname{sech}^{4}\left(k_{i-\frac{1}{2}}^{-}\left(-j v_{i-\frac{1}{2}}^{-} \tau-1\right)\right) \\
& +6 B_{i}^{3} k_{i}^{2} v_{i-\frac{1}{2}}^{-} \tau \tanh \left(k_{i}\left(1-j v_{i-\frac{1}{2}}^{-} \tau\right)\right) \operatorname{sech}^{4}\left(k_{i}\left(1-j v_{i-\frac{1}{2}}^{-} \tau\right)\right) \\
& -6 B_{i} \alpha \tanh \left(k_{i}\left(1-j v_{i-\frac{1}{2}}^{-} \tau\right)\right)+4 B_{i-\frac{1}{2}}^{-}{ }^{3} k_{i-\frac{1}{2}}^{-}{ }^{5} v_{i-\frac{1}{2}}^{-} \mu \tau^{2} \\
& \times \tanh ^{2}\left(k_{i-\frac{1}{2}}^{-}\left(-j v_{i-\frac{1}{2}}^{-} \tau-1\right)\right) \operatorname{sech}^{6}\left(k_{i-\frac{1}{2}}^{-}\left(-j v_{i-\frac{1}{2}}^{-} \tau-1\right)\right) \\
& +4 B_{i}^{3} k_{i}^{3} v_{i-\frac{1}{2}}^{-} \tau^{2} \tanh ^{2}\left(k_{i}\left(1-j v_{i-\frac{1}{2}}^{-} \tau\right)\right) \operatorname{sech}^{4}\left(k_{i}\left(1-j v_{i-\frac{1}{2}}^{-} \tau\right)\right) \\
& +12 B_{i}^{2} k_{i}^{3} v_{i-\frac{1}{2}}^{-} \mu \tau \tanh \left(k_{i}\left(1-j v_{i-\frac{1}{2}}^{-} \tau\right)\right) \operatorname{sech}^{4}\left(k_{i}\left(1-j v_{i-\frac{1}{2}}^{-} \tau\right)\right) \\
& -6 B_{i}^{2} \tanh ^{2}\left(k_{i}\left(1-j v_{i-\frac{1}{2}}^{-} \tau\right)\right) \\
& +4 B_{i}^{4} k_{i}^{4} v_{i-\frac{1}{2}}^{-2} \tau^{2} \tanh ^{2}\left(k_{i}\left(1-j v_{i-\frac{1}{2}}^{-} \tau\right)\right) \operatorname{sech}^{6}\left(k_{i}\left(1-j v_{i-\frac{1}{2}}^{-} \tau\right)\right) \\
& +8 B_{i}^{2} k_{i}^{4} v_{i-\frac{1}{2}}^{-2} \mu \tau^{2} \tanh ^{2}\left(k_{i}\left(1-j v_{i-\frac{1}{2}}^{-} \tau\right)\right) \operatorname{sech}^{4}\left(k_{i}\left(1-j v_{i-\frac{1}{2}}^{-} \tau\right)\right) \\
& +8 B_{i}^{3} k i^{5} v_{i-\frac{1}{2}}^{-}{ }^{2} \mu \tau^{2} \operatorname{sech}\left[k_{i}\left(1-j v_{i-\frac{1}{2}}^{-} \tau\right)\right]^{6} \tanh \left[k_{i}\left(1-j v_{i-\frac{1}{2}}^{-} \tau\right)\right]^{2} \\
& -12 B_{i} \tanh \left[k_{i-\frac{1}{2}}^{-}\left(x-j v_{i-\frac{1}{2}}^{-} \tau\right)\right]+12 B_{i} \tanh \left[k_{i}\left(x-(1+j) v_{i-\frac{1}{2}}^{-} \tau\right)\right]
\end{aligned}
$$

and where:

$$
C_{i}=-\int_{I_{i}} F P_{l_{x}}^{i}(x) d x
$$

$B_{i}, k_{i}, v_{i}, B_{i-\frac{1}{2}}^{-}, k_{i \pm \frac{1}{2}}^{ \pm}, v_{i \pm \frac{1}{2}}^{ \pm}$can take any values in $\mathbb{R}$.

The integral terms have been computed exactly.

In the same way, bell-profile solitary waves, or combinations of bell and kink-profile ones, could be obtained.

It thus exhibits the existence of lattice solitons, related to the discrete numerical scheme, of the form

$$
u_{i}^{n}=A_{i} \operatorname{Sech}\left[k_{i}\left(i h-n v_{i} \tau\right)\right]+B_{i} \operatorname{Tanh}\left[k_{i}\left(i h-n v_{i} \tau\right)\right], \quad\left(B_{i}, k_{i}, v_{i}\right) \in \mathbb{R}^{3}
$$

where $A_{i \pm 1}=A_{i \pm \frac{1}{2}}^{ \pm}, B_{i \pm 1}=B_{i \pm \frac{1}{2}}^{ \pm}$are linked to $A_{i}, B_{i}$ by means of the recursive relation (41).

Figure 1 displays a lattice solitary wave, first, for $\sigma=0.7, h=0.01, v=5, k=5$, as a function of the mesh points. 
Fig. 1 A lattice solitary wave, first, for $\sigma=0.7, h=0.01$, $v=5, k=5$, as a function of the mesh points

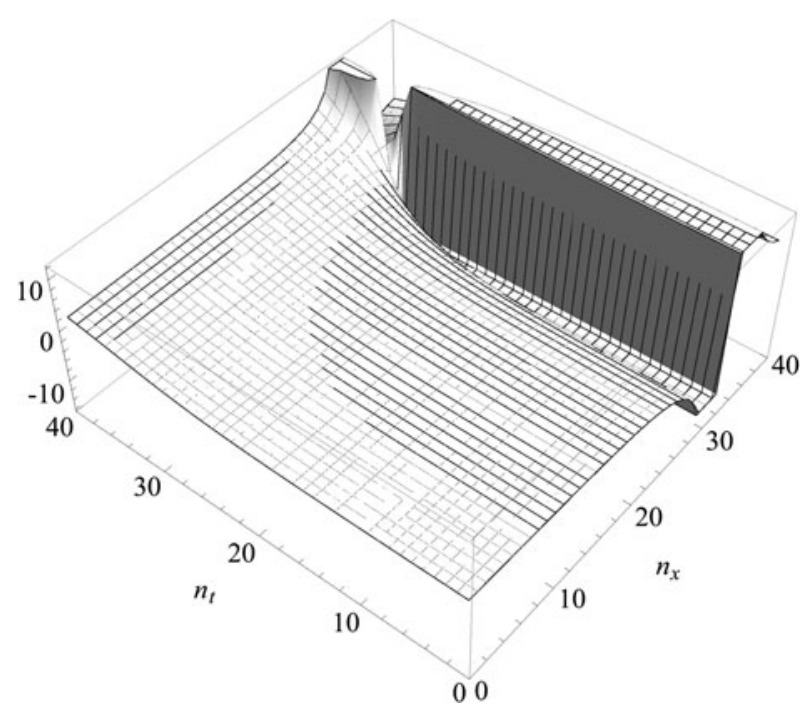

4.2 The Case of the HLL Flux, for the One-Dimensional Euler Equations

Using (36), the solution is searched, for each cell $I_{i}$, corresponding to $x=i h$, at the time $t=n \tau$, under the form:

$$
u^{H L L}= \begin{cases}u^{-} & \text {if } 0 \leqslant s^{-} \\ u_{L}^{*} & \text { if } s^{-} \leqslant 0 \leqslant s^{+} \\ u^{+} & \text {if } s^{+} \leqslant 0\end{cases}
$$

As previously, we set:

$$
\begin{aligned}
& \left\{\begin{array}{c}
u_{i}^{ \pm}=A_{i}^{ \pm} \operatorname{sech}\left[k_{i}^{ \pm}\left(i h-n v_{i}^{ \pm} \tau\right)\right]+B_{i}^{ \pm} \tanh \left[k_{i}^{ \pm}\left(i h-n v_{i}^{ \pm} \tau\right)\right] \\
u^{ \pm}{ }_{i \pm \frac{1}{2}}^{ \pm}=A^{ \pm}{ }_{i \pm \frac{1}{2}}^{ \pm} \operatorname{sech}\left[k^{ \pm}{ }_{i \pm \frac{1}{2}}^{ \pm}\left(i h-n v^{ \pm}{ }_{i \pm \frac{1}{2}}^{ \pm} \tau\right)\right] \\
+B^{ \pm}{ }_{i \pm \frac{1}{2}}^{ \pm} \tanh \left[k^{ \pm}{ }_{i \pm \frac{1}{2}}^{ \pm}\left(i h-n v^{ \pm}{ }_{i \pm \frac{1}{2}}^{ \pm} \tau\right)\right]
\end{array}\right. \\
& \left\{\begin{array}{c}
u_{L_{i}}^{*}=A_{L_{i}}^{*} \operatorname{sech}\left[k_{L_{i}}^{*}\left(i h-n v_{L_{i}}^{*} \tau\right)\right]+B_{L_{i}}^{*} \tanh \left[k_{L_{i}}^{*}\left(i h-n v_{L_{i}}^{*} \tau\right)\right] \\
u_{L_{i \pm \frac{1}{2}}}^{*}=A_{L_{i \pm \frac{1}{2}}}^{*} \operatorname{sech}\left[k_{L_{i \pm \frac{1}{2}}^{*}}^{*}\left(i h-n v_{L_{i \pm \frac{1}{2}}}^{*} \tau\right)\right] \\
+B_{L_{i \pm \frac{1}{2}}^{*}}^{*} \tanh \left[v_{L_{i \pm \frac{1}{2}}^{*}}^{*}\left(i h-n v_{L_{i \pm \frac{1}{2}}}^{*} \tau\right)\right]
\end{array}\right.
\end{aligned}
$$

The constants $A_{i}^{ \pm}, B_{i} \pm, k_{i}^{ \pm}, v_{i}^{ \pm}, A^{ \pm}{ }_{i \pm \frac{1}{2}}^{ \pm}, B^{ \pm}{ }_{i \pm \frac{1}{2}}^{ \pm}, k^{ \pm}{ }_{i \pm \frac{1}{2}}^{ \pm}, v^{ \pm}{ }_{i \pm \frac{1}{2}}^{ \pm}, A_{L_{i}^{*}}^{*}, B_{L_{i}}^{*}, k_{L_{i}}^{*}, v_{L_{i}^{*}}$, $A_{L_{i \pm \frac{1}{2}}}^{*}, B_{L_{i \pm \frac{1}{2}}}^{*}, k_{L_{i \pm \frac{1}{2}}}^{*}, v_{L_{i \pm \frac{1}{2}}^{*}}^{*}$, naturally depend on the $i^{t h}$ cell. Depending on their existence, and of the one of integers $i, n$ satisfying this relation, spurious lattice solitary waves will or not appear.

For sake of simplification, we also set:

$$
\left\{\begin{array}{l}
\mathcal{E}^{ \pm}=\left\{A_{i}^{ \pm}, B_{i}^{ \pm}, k_{i}^{ \pm}, v_{i}^{ \pm}\right\} \\
\mathcal{E}_{L}^{*}=\left\{A_{L_{i \pm \frac{1}{2}}^{*}}^{*}, B_{L_{i \pm \frac{1}{2}}^{*}}^{*}, k_{L_{i \pm \frac{1}{2}}^{*}}^{*}, v_{L_{i \pm \frac{1}{2}}^{*}}^{*}\right\}
\end{array}\right.
$$


Replacing (45), (35) in (24), for the first Legendre polynomial, in the specific case of kink profile solitary waves, for which the sech constants are taken equal to zero $\left(A_{i}^{ \pm}=A_{L_{i}^{*}}^{*}=\right.$ $A_{i \pm \frac{1}{2}}^{ \pm}=A_{L_{i \pm \frac{1}{2}}^{ \pm}}^{*}=0$ ), one obtains:

$$
\left\{\begin{array}{l}
B^{ \pm}{ }_{i+\frac{1}{2}}^{+}=-6 \alpha \tanh \left(k_{i+\frac{1}{2}}^{ \pm}\left(1-j v_{i+\frac{1}{2}}^{ \pm} \tau\right)\right) \mathcal{B}\left(\mathcal{E} \backslash B_{i+\frac{1}{2}}^{ \pm}, C_{i} \pm\right) \\
B_{L_{i+\frac{1}{2}}^{*}}^{*}=-6 \alpha \tanh \left(k_{L_{i+\frac{1}{2}}^{*}}^{*}\left(1-j v_{L_{i+\frac{1}{2}}^{*}}^{+ \pm}\right)\right) \mathcal{B}\left(\mathcal{E} \backslash B_{L_{i+\frac{1}{2}}^{+}}^{*}, C_{i}^{*}\right)
\end{array}\right.
$$

where the function $\mathcal{B}$ is given by (42), with:

$$
\left\{\begin{array}{l}
C_{i}^{ \pm}=-\int_{I_{i}} F^{ \pm} P_{l x}^{i}(x) d x \\
C_{i_{L}^{*}}^{*}=-\int_{I_{i}} F_{l}^{*} P_{l x}^{i}(x) d x
\end{array}\right.
$$

The integral terms have been computed exactly.

In the same way, bell-profile solitary waves, or combinations of bell and kink-profile ones, could be obtained.

It thus exhibits the existence of lattice solitons, related to the discrete numerical scheme, of the form

$$
\begin{aligned}
& \left\{\begin{array}{c}
u_{i}^{ \pm}=A_{i}^{ \pm} \operatorname{sech}\left[k_{i}^{ \pm}\left(i h-n v_{i}^{ \pm} \tau\right)\right]+B_{i}^{ \pm} \tanh \left[k_{i}^{ \pm}\left(i h-n v_{i}^{ \pm} \tau\right)\right] \\
u^{ \pm}{ }_{i \pm \frac{1}{2}}^{ \pm}=A^{ \pm}{ }_{i \pm \frac{1}{2}}^{ \pm} \operatorname{sech}\left[k^{ \pm}{ }_{i \pm \frac{1}{2}}{ }^{ \pm}\left(i h-n v^{ \pm}{ }_{i \pm \frac{1}{2}}{ }^{ \pm} \tau\right)\right] \\
+B^{ \pm}{ }_{i \pm \frac{1}{2}}^{ \pm} \tanh \left[k^{ \pm}{ }_{i \pm \frac{1}{2}}{ }^{ \pm}\left(i h-n v^{ \pm}{ }_{i \pm \frac{1}{2}}^{ \pm} \tau\right)\right]
\end{array}\right.
\end{aligned}
$$

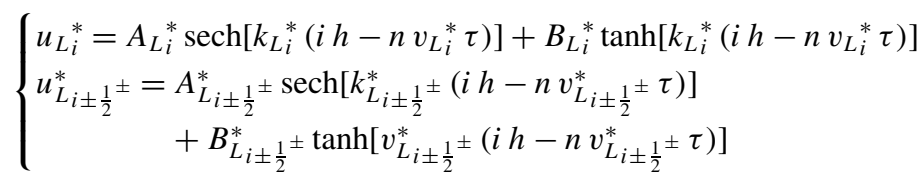

where $B_{i+\frac{1}{2}}^{ \pm}, B_{L_{i+\frac{1}{2}}}^{*}$ are linked to $\mathcal{E} \backslash B_{i+\frac{1^{2}}{+}}^{ \pm}, \mathcal{E}_{L} \backslash B_{L_{i+\frac{1}{2}}}^{*}$ by means of the recursive relation (49), where $\left(\mathcal{E} \backslash B_{i+\frac{1}{2}}^{ \pm}, \mathcal{E}_{L} \backslash B_{L_{i+\frac{1}{2}}}^{*}\right) \in \mathbb{R}^{7} \times \mathbb{R}^{7}$.

\section{Concluding Remarks}

The existence of spurious numerical lattice solitary waves for discontinuous Galerkin schemes has been proved, for different kind of fluxes, as the Lax-Friedrichs and HLL fluxes. In the same way, the existence of spurious solitary waves for other fluxes could be shown. Such lattice solitary waves, which are not solutions of the exact continuous original equation, nevertheless satisfy the numerical scheme, appearing as parasitic solutions of the correct one. Such schemes will be referred to as structurally instable ones. Such spurious solitary waves have constant energy, and therefore the numerical error norm does not vanish at arbitrary long integration times on unbounded numerical domains.

\section{References}

1. David, C., Sagaut, P.: Structural stability of finite dispersion-relation preserving schemes. Chaos Solitons Fractals 41(4), 2193-2199 (2009) 
2. David, C., Sagaut, P.: Spurious solitons and structural stability of finite difference schemes for nonlinear wave equations. Chaos Solitons Fractals 41(2), 655-660 (2009)

3. Reed, W., Hill, T.: Triangular mesh methods for neutron transport equation. Tech. Report LA-UR-73479, Los Alamos Scientific Laboratory (1973)

4. Lesaint, P., Raviart, P.: On a finite element method for solving the neutron transport equation. In: de Boor, C (ed.) Mathematical Aspects of Finite Elements in Partial Differential Equations, pp. 89-145. Academic Press, San Diego (1974)

5. Lin, Q., Zhou, A.: Convergence of the discontinuous Galerkin method for a scalar hyperbolic equation. Acta Math. Sci. 13, 291-298 (1993)

6. Lin, Q., Zhou, A.: Some arguments for recovering the finite element error of hyperbolic problems. Acta Math. Sci. 11, 471-476 (1991)

7. Lin, Q., Zhu, Q.: The Preprocessing and Postprocessing for the Finite Element Methods. Shanghai Sci. \& Tech., Shanghai (1994)

8. Cockburn, B., Shu, C.-W.: TVB Runge-Kutta local projection discontinuous Galerkin finite element method for conservation laws II: General framework. Math. Comput. 52, 411-435 (1989)

9. Cockburn, B., Shu, C.-W.: Runge-Kutta discontinuous Galerkin methods for convection-dominated problems. J. Sci. Comput. 16, 173-261 (2001)

10. Cockburn, B., Karniadakis, G.E., Shu, C.-W. (eds.): Discontinuous Galerkin Methods. Springer, Berlin (2000)

11. Feistauer, M., Švadlenka, K.: Discontinuous Galerkin method of lines for solving nonstationary singularly perturbed linear problems. J. Numer. Math. 12(2), 97-117 (2004)

12. Houston, P., Jensen, M., Süli, E.: HP-discontinuous Galerkin finite element methods with least-squares stabilization. J. Sci. Comput. Arch. 17(1-4), 3-25 (2002)

13. Atkins, H., Shu, C.-W.: Quadrature-free implementation of discontinuous Galerkin methods for hyperbolic equations. AIAA J. 36, 775 (1998)

14. Burgers, J.M.: Mathematical examples illustrating relations occurring in the theory of turbulent fluid motion. Trans. R. Neth. Acad. Sci. Amsterdam 17, 1-53 (1939)

15. Feng, Z., Chen, G.: Solitary wave solutions of the compound burgers-Korteweg-de Vries equation. Physica A 352, 419-435 (2005)

16. David, C., Fernando, R., Feng, Z.: A note on "general solitary wave solutions of the compound BurgersKorteweg-de Vries Equation. Phys. A, Stat. Theor. Phys. 375(1), 44-50 (2007)

17. Giraldo, F.X., Hesthaven, J.S., Warburton, T.: Nodal high-order discontinuous Galerkin methods for the spherical shallow water equations. J. Comput. Phys. Arch. 181(2), 499-525 (2002)

18. Harten, A., Lax, P.D., van Leer, B.: On upstream differencing and Godunovtype schemes for hyperbolic conservation laws. SIAM Rev. 25, 35-61 (1983)

19. Toro, E.F.: Riemann Solvers and Numerical Methods for Fluid Dynamics, A Practical Introduction. Springer, Berlin (1997)

20. Whitham, G.B.: Linear and Nonlinear Waves. Wiley, New York (1974)

21. Dodd, R.K., Eilbeck, J.C., Gibbon, J.D., Morris, H.C.: Solitons and Nonlinear Wave Equations. Academic Press, London (1983)

22. Johnson, R.S.: A Modern Introduction to the Mathematical Theory of Water Waves. Cambridge University Press, Cambridge (1997)

23. Ince, E.L.: Ordinary Differential Equations. Dover, New York (1956)

24. Birkhoff, G., Rota, G.C.: Ordinary Differential Equations. Wiley, New York (1989)

25. Polyanin, A.D., Zaitsev, V.F.: Handbook of Nonlinear Partial Differential Equations. Chapman and Hall/CRC, London (2004) 